ArtefaCToS. Revista de estudios de la ciencia y la tecnología

eISSN: $1989-3612$

Vol. 9, No. 2 (2020), 2. ${ }^{\text {Época, 5-26 }}$

DOI: https://doi.org/10.14201/art202092526

\title{
La cientifización de la raza: el carácter pseudocientífico de las disciplinas
}

\section{The Scientificization of Race: The Pseudoscientific Character of the Disciplines}

\section{Celia MARTÍNEZ GONZÁLEZ}

Universidad de Oviedo, España

mgonzalez.celia@gmail.com

Recibido: 25/09/2020. Revisado: 12/10/2020. Aceptado: 14/10/2020

\section{Resumen}

La naturalización de la raza y los intentos por parte de ciertas disciplinas científicas de elaborar una jerarquización racial forman parte de la historia de la ciencia. En este artículo se analizan disciplinas que pertenecen al denominado "racismo científico" para justificar su carácter pseudocientífico tanto desde una perspectiva diacrónica como desde una perspectiva sincrónica, prestando especial atención a la utilización que en ellas se hace de un concepto esencialista de raza. En primer lugar, se abordan criterios para identificar las pseudociencias, así como rasgos que estas presentan. A continuación, se someten a análisis las disciplinas objeto de estudio, incidiendo en los momentos de transición de unas a otras. Tras ello se concluye que las disciplinas estudiadas fueron afectadas por prejuicios y una fuerte ideología racial que repercuten directamente en su estatus pseudocientífico.

Palabras clave: jerarquía racial; demarcación de la ciencia; pseudociencia; frenología; esencialismo; objetividad.

\begin{abstract}
The naturalization of race and attempts by certain scientific disciplines to develop a racial hierarchy are part of the history of science. This paper analyses a number of disciplines that belong to the so-called "scientific racism" in order to justify its pseudo-scientific character both from a diachronic perspective and from a synchronic perspective, paying special attention to the development of
\end{abstract}


an essentialist concept of race. First, criteria are addressed to identify pseudosciences, as well as features that these present. Next, the disciplines under study are analyzed, focusing on the moments of transition from one to the other. After that, it is concluded that the disciplines studied were affected by prejudices and a strong racial ideology that directly affect their pseudoscientific status.

Keywords: racial hierarchy; science demarcation; pseudoscience; phrenology; essentialism; objectivity.

\section{Introducción}

En el momento actual, numerosas disciplinas científicas ampliamente desarrolladas en el pasado y que gozaron de prestigio son consideradas pseudocientíficas. En este artículo se analizan cinco disciplinas en el periodo de tiempo que abarca desde 1820 hasta 1920: la frenología, la craneometría, la demografía racial, la antropología criminal y las pruebas de medición de inteligencia. La focalización del estudio concretamente en estas disciplinas responde a la identificación de una conexión especialmente relevante entre todas ellas: la utilización de un concepto esencialista de raza. Aun no siendo el aspecto más destacado de las teorías desarrolladas por esas disciplinas, la raza desempeña un papel prácticamente central en ellas, pues desde todas se pretendió elaborar una jerarquía racial natural. Uno de los objetivos de esta investigación, si no el objetivo principal, es mostrar cómo la utilización de este concepto esencialista de raza contribuye de manera determinante a la pseudocientificidad de las disciplinas. Asimismo, el análisis de este concepto de raza facilita la observación de una serie de creencias o hipótesis presentes en todas las disciplinas y que están íntimamente relacionadas con una de las cuestiones centrales de esta investigación: la presencia de prejuicios en la investigación científica.

En lo que sigue se defenderá que estas disciplinas son pseudocientíficas precisamente a causa de las connotaciones del concepto esencialista de raza utilizado. Se descarta que estas disciplinas ejemplifiquen casos de mala ciencia, esto es, que la ciencia racista sea el resultado de una mala aplicación del método científico, tal y como se defendería desde un empirismo feminista de corte más clásico, de acuerdo con Amparo Gómez Rodríguez (2004). Aunque se prestará atención a aspectos metodológicos de cada una de las disciplinas, la principal problemática observada al respecto de la raza responde a la objetividad científica y a los valores externos que son introducidos en la ciencia. Esos valores, en este caso concreto, racistas son dependientes de la sociedad y del contexto en el que se insertan y son introducidos por los propios científicos que, en los casos que se expondrán, son plenamente conscientes de que están introduciendo una ideología de raza con el fin de preservar cierto statu quo y naturalizar prejuicios y creencias arraigados en la sociedad. 
Es menester para poder llegar a estas conclusiones plantear en primer lugar una serie de criterios o pautas para identificar las prácticas pseudocientíficas. A ello seguirá el análisis pertinente de cada una de las disciplinas, prestando especial atención a las transiciones entre ellas.

\section{Criterios de demarcación y rasgos de las pseudociencias}

La cuestión de la demarcación remite a una problemática de suma importancia: distinguir entre lo que es científico y lo que no es científico. Es preciso, sin embargo, tener presente que lo "no científico" engloba la pseudociencia, la ciencia fronteriza y también los conocimientos no científicos en términos generales. Desde una perspectiva teórica, la demarcación es útil para nuestra disciplina, la Filosofía de la ciencia, pero también para la propia ciencia. Desde una perspectiva práctica, la importancia de la demarcación tiene que ver con la aplicación de la ciencia, pero también con la investigación científica. Michael Shermer (2013, 206) expone muy bien la importancia de la cuestión práctica diferenciando entre ciencia normal, pseudociencia y ciencia fronteriza, especificando la provisionalidad de la membresía a esas categorías. Las disciplinas que se toman como objeto de estudio y de reflexión en este trabajo se caracterizan por haber sido consideradas científicas en una época concreta o, en su defecto, por haber sido presentadas como científicas por aquellos que las practicaban. Aunque en la actualidad son consideradas de manera unánime como pseudociencias, cabe preguntarse a qué se debe ese cambio de estatus.

La investigación sobre el denominado "problema de la demarcación" fue abandonada mayoritariamente tras la declaración de intenciones de Larry Laudan en su artículo "The Demise of the Demarcation Problem" (1983), tal y como recogen prácticamente todos los autores que abordan en la actualidad la cuestión. Tras un extenso análisis de lo que él denomina "tradición demarcacionista" concluye que preguntarse por las razones de la cientificidad de las creencias no es ni interesante ni tratable, y aboga por deshacerse de términos como "pseudociencia”, pues son simplemente recursos retóricos que no aportan nada a la investigación empírica (Laudan, 1983, 125).

No hay duda de que este texto de Laudan contribuyó al abandono de la investigación sobre la demarcación, investigación caracterizada por la multiplicidad de enfoques y perspectivas. Sin embargo, ¿̨tiene razón Laudan al decir que los términos como "pseudociencia" y similares son simplemente recursos retóricos? En este epígrafe se propone rebatir la afirmación categórica de Laudan y mostrar que esos términos son necesarios para poder argumentar y reflexionar sobre la ciencia, tanto desde "dentro" de la ciencia, como desde disciplinas que la estudien.

\subsection{La propuesta de S. O. Hansson: tres criterios de demarcación}

Ediciones Universidad de Salamanca / @@ब

ArtefaCToS, Vol. 9, No. 2 (2020), 2. a Época, 5-26 
Hansson sostiene que la gran cantidad de criterios ofrecidos para resolver la cuestión de la demarcación se debe a que esos criterios fueron buscados en el nivel equivocado de especificidad epistemológica $(2013,62)$. Aboga por una observación de la interconexión existente entre ciencias naturales y humanidades, la cual servirá para articular la demarcación. La tarea de distinguir entre ciencia y pseudociencia requiere tener en cuenta, como se indicó anteriormente, que no es lo mismo "pseudocientífico" que "no-científico". Por ello, es preciso revisar lo que se entiende por pseudociencia para poder proponer unos criterios específicos de demarcación ciencia/pseudociencia, que es, en última instancia, lo que se busca, y no demarcar la ciencia con respecto al resto de prácticas y disciplinas que son no-científicas. En primer lugar, por lo tanto, formula un criterio que recoge el hecho de que la pseudociencia se inserta en el dominio de la ciencia: el criterio de dominio científico.

Desde la perspectiva internalista de Hansson, la pseudociencia no se caracteriza únicamente por no ser ciencia, sino porque se desvía de lo que consideramos ciencia. Una manera de evaluar esta desviación es atendiendo a los criterios de calidad de la ciencia. Esos criterios son los siguientes: fiabilidad, fertilidad y utilidad $(2013,66)$. Están interrelacionados entre sí, sin embargo, solo uno es relevante de cara a nuestro interés: la fiabilidad. Hansson la define de la siguiente manera: "un enunciado científico debería ser correcto o, más bien, lo más cercano a la corrección que se pueda lograr actualmente" (Hansson, 2013, 66). Fertilidad y utilidad son dos criterios de calidad que en ocasiones no son satisfechos y no por ello las investigaciones que no los satisfacen son pseudociencia. La fiabilidad, sin embargo, es el criterio de calidad más básico, por lo que, si ese criterio no se da en la investigación sí estaremos ante un caso de pseudociencia. Una vez se concluye que la pseudociencia se caracteriza por no satisfacer un criterio de calidad tan básico como es la fiabilidad, Hansson formula el siguiente criterio de demarcación: el criterio de no-fiabilidad, que es condición necesaria en la definición de pseudociencia.

Además, las pseudociencias se caracterizan por ofrecer una imagen de cientificidad o por ser calificadas como científicas por aquellos que las practican. Esta pretensión de cientificidad, de mostrarse como una supuesta doctrina científica, es para Hansson, sin embargo, un requisito demasiado amplio para considerarlo útil. Sostiene que el elemento que verdaderamente caracteriza a la pseudociencia es la "doctrina desviada". Este criterio recoge la pretensión por parte de quienes desarrollan prácticas pseudocientíficas de presentarlas "como el conocimiento más fiable en su tema” (2013, 70). Por tanto, para Hansson, un enunciado es pseudocientífico si y solo si satisface los tres siguientes criterios:

i. Es propio de un problema dentro de los dominios de la ciencia en sentido amplio (el criterio de dominio científico).

ii. Sufre de una falta de fiabilidad tan severa que no puede ser fiable (el criterio de no-fiabilidad). 
iii. Es parte de una doctrina cuyos principales proponentes tratan de crear la impresión de que representa el conocimiento más fiable en su tema (el criterio de doctrina desviada) $(2013,70)$.

Si bien de la propuesta de Hansson se puede decir que es un tanto ambigua o incluso demasiado generalista, parece claro que el propio autor asume esa dificultad cuando reconoce que se trata de una definición exhaustiva, pero no operacional o directamente aplicable a causa de la no-uniformidad de las ciencias y de los cambios que estas experimentan. Y esta última apreciación es especialmente importante, pues Hansson concibe la ciencia como una actividad situada histórica y culturalmente, por lo que el grado de pseudocientificidad de las disciplinas es susceptible de cambio.

\subsection{Revisión de otros rasgos de las pseudociencias}

Por otro lado, resulta interesante atender a aportaciones que han hecho otros autores sobre la cuestión de las pseudociencias para poder ofrecer un análisis más profundo. Pero se debe precisar que las características que serán expuestas a continuación no se aplican necesariamente a todas las disciplinas pseudocientíficas y, por lo tanto, no serán encontradas en todos los casos estudiados en este trabajo. Estas características se desligan de los análisis históricos que se hacen de las pseudociencias y de la propia ciencia y resultan útiles tanto para comprender el propio concepto de pseudociencia como para comprender la importancia de distinguir qué es pseudocientífico. Los tres rasgos más relevantes para esta investigación y que seguidamente serán expuestos son los siguientes: la relación de las pseudociencias con la práctica científica, los sistemas de creencia pseudocientíficos y la autoridad epistémica.

\subsubsection{Las pseudociencias y la práctica científica: difusión y popularización}

El periodo de desarrollo de las disciplinas aquí estudiadas comprende desde 1820, año en que se funda la Sociedad Frenológica de Edimburgo, hasta 1920. La elección de esta última fecha es meramente orientativa y responde a la necesidad de delimitar el periodo de estudio; ahora bien, garantiza la inclusión de los primeros desarrollos en las pruebas de medición de la inteligencia en los Estados Unidos de América. La Historia de la ciencia muestra que la profesionalización de la ciencia alcanza su punto álgido en el siglo XX, pero en las primeras décadas del siglo XIX no estaba muy claro qué era exactamente la ciencia. Daniel P. Thurs y Ronald L. Numbers $(2013,125)$ recalcan este hecho cuando afirman que "en el segundo cuarto de 1800s (...) la ciencia iba más allá de lo natural e incluía una enorme franja de áreas, desde la teología a la taquigrafía”. Evaluar el carácter pseudocientífico de una disciplina demanda tener en cuenta el factor histórico y analizar la ciencia en su momento histórico correspondiente. Ahora

Ediciones Universidad de Salamanca / @@ब

ArtefaCToS, Vol. 9, No. 2 (2020), 2. a Época, 5-26 
bien, incorporar una perspectiva histórica al estudio de las disciplinas y doctrinas pseudocientíficas no está reñido con ser crítico con esas disciplinas. La frenología, por ejemplo, recibió acusaciones tempranas de ser una pseudociencia (el primer uso formal del término "pseudociencia" tuvo lugar en 1824 y fue dirigido a la frenología, de acuerdo con Thurs y Numbers $(2013,125))$, pero no era una opinión mayoritaria. No fue hasta las últimas décadas del siglo XIX cuando fue considerada como tal. Y, precisamente, las décadas en las que la frenología se encontraba en ese estado de indeterminación son las décadas en las que mayor auge tuvo, como recoge Sysling $(2018,266)$.

A finales del siglo XIX comienza a haber un consenso generalizado sobre los rasgos que caracterizaban a las pseudociencias, siendo el principal el método defectuoso del que se valían, llegando a conclusiones sin tener en cuenta la evidencia empírica. Pero también se llamaba la atención sobre la mercantilización que se asociaba con todas las disciplinas pseudocientíficas. Sin embargo, mientras que el primer rasgo sí puede ser generalmente aplicado a las disciplinas que hoy en día son generalmente consideradas pseudocientíficas, no sucede lo mismo con el segundo rasgo. De los casos analizados en este trabajo solamente uno, la frenología, presentaba esa vertiente "comercial".

La reflexión sobre la relación entre la pseudociencia y la ciencia en esas décadas en las que la línea que las separa era un tanto borrosa debido a que la ciencia aún no se ha profesionalizado completamente obliga a prestar atención a la difusión de las diferentes disciplinas. Los medios de comunicación siempre han sido una plataforma de la que las disciplinas se han valido para transmitir su cuerpo de conocimientos o creencias. En la actualidad esto sigue siendo así, tanto en el caso de las ciencias (se dedican espacios especializados en la comunicación científica) como en el caso de las pseudociencias (prácticamente en la totalidad de prensa hay un espacio dedicado al horóscopo, un método de la astrología). En su momento de auge, los frenólogos se anunciaban en la prensa, pero también lo hacían los científicos y otra serie de practicantes de pseudociencias (astrólogos, psíquicos...).

La popularización de la ciencia se llevó a cabo a través de prensa especializada en la que los científicos del momento exponían sus teorías o planteaban su visión del mundo, pero la prensa generalista también recogía los debates científicos más candentes del momento (Gregory y Miller, 1998, 24). La comunicación científica era esencial para dotar a las masas de un conocimiento general científico que, además, reafirmara valores sociales o políticos del momento. Esta popularización de la ciencia era complementada con la organización de conferencias, normalmente accesibles al público general y no solamente dedicadas al público especializado. Están ampliamente documentadas, por ejemplo, las giras que hicieron por Europa tanto Franz Joseph Gall (van Wyhe, 2004; Sakalauskaité-Juodeikiené, Eling, y Finger, 2017; Thomassen y Beierholm, 2020) como su discípulo Johann Spurzheim (Cantor, 1975; Kaufman, 1998; van Wyhe, 2004). Mas el acceso 
del público a la ciencia también se llevaba a cabo a través de los gabinetes que los científicos utilizaban como muestrarios: así lo hizo, por ejemplo, Morton (Fabian, 2010), quien dispuso un gabinete-museo en el que exponía todos los especímenes (cráneos) que había ido coleccionando a lo largo de los años.

\subsubsection{Sistemas de creencia pseudocientíficos}

Erich Goode acepta la idea de pseudociencia como doctrina desviada de Hansson. Su interés reside principalmente en estudiar la pseudociencia desde una perspectiva sociológica, pues una característica de las pseudociencias es la conexión que establecen con la sociedad.

Así, Goode (2013, 147-150) establece cinco tipos de sistemas de creencia pseudocientíficos:

i. Creencias que dependen de la relación cliente-practicante.

ii. Sistemas de creencia insertos en una tradición religiosos y sostenidos por una institución religiosa.

iii. Creencias en las se practica la forma, pero no el contenido de la ciencia

iv. Sistemas de creencia sostenidos por un público amplio.

v. Sistemas de creencia originados individualmente.

De los cinco sistemas que propone Goode interesa en particular el primero de ellos: creencias que dependen de la relación cliente-practicante. El caso de la frenología es el más claro a este respecto: las mediciones frenológicas requerían de una confianza por parte del cliente en el practicante, que estaba rodeado de una suerte de aura de autoridad epistémica, por ser el especialista.

Si bien se ha calificado a la frenología como un sistema de creencias en el que prima la relación cliente-practicante debido a la vertiente "clínica" de la disciplina, se ha de tener en cuenta que también puede ser considerada (al igual que el resto de las disciplinas que expondremos) como un sistema de creencias sostenidos por un público amplio. Las disciplinas exploradas en este trabajo son disciplinas que se caracterizan por haber tenido un respaldo muy amplio en su momento por parte de la comunidad científica. Por ejemplo, en el caso de las pruebas desarrolladas para medir la inteligencia sucede que la creencia específica en esa disciplina es que la inteligencia es una variable, presuntamente independiente, susceptible de ser medida. Pero la creencia básica común a todas las disciplinas es que existen razas humanas y que estas pueden ser jerarquizadas "científicamente".

\subsubsection{Autoridad epistémica}

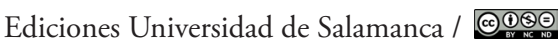

ArtefaCToS, Vol. 9, No. 2 (2020), 2. a Época, 5-26 
Estrechamente relacionada con el punto anterior, la cuestión de la autoridad epistémica es un tanto problemática, pues la autoridad epistémica también se da en la ciencia. Veamos cómo podemos caracterizar la autoridad epistémica y cómo se ejercitaba esta desde las disciplinas analizadas.

Se ha de tener en cuenta, en primer lugar, que la autoridad epistémica puede ser interna o externa. En el caso de la autoridad epistémica interna, esta se ejerce sobre la comunidad científica. La autoridad epistémica externa es, por otro lado, aquella que se ejerce sobre aquello que no pertenece al ámbito científico.

La autoridad epistémica, especialmente la externa, tiene que ver con la difusión de los contenidos científicos y de la disposición de las masas a asumir esos contenidos. En algunos autores (Thurs y Numbers, 2013, 129) se identifica como "credulidad" la actitud de las masas en ciertos momentos con respecto a lo que ahora es reconocido como pseudociencias. La discusión sobre si es justo o pertinente hablar de credulidad se escapa del ámbito de este trabajo, pero enjuiciar prácticas ya superadas exige un ejercicio de historización también aplicable a este aspecto, por ello se rechaza calificar de credulidad la aceptación por parte de la sociedad normal de estas doctrinas, teniendo en cuenta, además, el acceso general a una educación amplia o, incluso, básica.

\section{Análisis de las disciplinas}

Hasta ahora se han expuesto los diferentes criterios a partir de los que se puede juzgar desde una perspectiva diacrónica el carácter pseudocientífico de las disciplinas. Además, se han identificado una serie de rasgos o características (por ejemplo, la autoridad epistémica) reconocibles en todas o en parte de las disciplinas. A continuación, se procederá a la exposición y análisis de las mismas para evaluar desde una perspectiva sincrónica las transiciones entre ellas y la incorporación o eliminación de contenidos de unas disciplinas con respecto a las demás. La transición de una disciplina a otra resulta de la incapacidad de las disciplinas para responder a las cuestiones planteadas, dando lugar a una transferencia de hipótesis y creencias que se incorporan a otra disciplina que, partiendo de unos métodos y materiales de investigación diferentes, busca responder a esas cuestiones. Al centrar la investigación en el concepto de raza, se observa cómo en el intento de justificar la realidad biológica de la raza y la legitimidad de establecer una jerarquía racial cada disciplina alcanza un punto límite y esa disciplina es reemplazada por otra que carga con la autoridad en la materia.

En este epígrafe, se exponen en orden cronológico las siguientes disciplinas: frenología, craneometría, demografía racial, antropología criminal y pruebas de medición de la inteligencia. Tal orden cronológico facilita el análisis sincrónico pretendido sobre la pseudocientificidad de las disciplinas. Se observará que estas difieren en métodos y teorías, pero el concepto esencialista de raza es común. 
Precisamente, la revisión de la trayectoria de ese concepto de raza a través de las disciplinas aporta evidencia que da más soporte al diagnóstico de la pseudocientificidad de estas.

Entre las disciplinas se distinguen aquellas que apelan a parámetros visibles, físicos (frenología, craneometría, antropología criminal y demografía racial) y aquellas que apelan a parámetros no visibles (las pruebas de medición de la inteligencia). En el caso de las primeras, los parámetros permanecen considerablemente estables a lo largo en el tiempo, siendo los principales aquellos que hacen referencia a las dimensiones craneales (por ejemplo, el índice cefálico). Las segundas, sin embargo, suponen un cambio radical en la cientifización de la raza, pues medirían la inteligencia, una entidad (tal y como la concebían los psicólogos que desarrollaron las pruebas que serán expuestas) no accesible por observación directa.

\subsection{Frenología}

La frenología, cuyo origen remite a los estudios de Franz Joseph Gall sobre la relación entre la forma del cráneo y el cerebro, fue una disciplina que gozó de mucha popularidad en el siglo XIX. En la teoría organológica de Gall, el cerebro era descrito como el órgano de la mente, el cual, a su vez, estaría conformado por órganos menores dedicado cada uno de ellos a una función específica. Se daría, además, una relación de proporcionalidad directa entre el tamaño relativo de cada órgano y su poder o fuerza, siendo esto visible a través de la observación del cráneo, cuya forma estaría determinada por la forma de los órganos del cerebro. Sin embargo, si algo caracteriza a la frenología y explica en gran parte la popularidad es la vertiente social de la disciplina introducida por Johann Gaspar Spurzheim, discípulo de Gall. Mientras que Gall consideraba su teoría como una teoría de la mente ligada a la psicología humana, Spurzheim buscó explotar la aplicación social de la teoría (Lyons, 1998, 493). Spurzheim fue el principal responsable del auge de la frenología en Reino Unido, tras haber dejado de trabajar junto a Gall e iniciar su gira de conferencias por ese país (Lyons, 1998; van Wyhe, 2004; Whitaker y Jarema, 2017).

La frenología fue acusada de ser una pseudociencia desde sus inicios: la lucha entre los detractores y los practicantes de la frenología ha sido bien plasmada por Cantor (1975), quien recopila críticas formuladas tanto contra aspectos teóricos como metodológicos. Por ejemplo, en 1815 fue "etiquetada como 'pura charlatanería' y sus practicantes como 'empíricos' deshonestos" (199, cursivas del autor). De un tono similar es una crítica de 1835 recogida por Sakalauskaité-Juodeikiené, Eling y Finger $(2017,400)$ en la que la frenología era considerada una "teoría no científica, que podría ser comparada con la astrología".

Sin embargo, pese a las críticas, la frenología experimentó un auge importante en las primeras décadas del siglo XIX. La investigación de Steven Shapin (1975) 
sobre la relevancia de la dimensión social en el desarrollo y afianzamiento de la frenología constituye una de las más importantes referencias para comprender ese auge. En esa época numerosas sociedades frenológicas fueron fundadas por todo el mundo, siendo la primera y la más destacada la Sociedad Frenológica de Edimburgo, fundada por los hermanos Combe. La frenología, presentada como una disciplina capaz de determinar las aptitudes psicológicas de los individuos a través del examen del cráneo, fue uno de los grandes atractivos de la sociedad victoriana. Las sociedades frenológicas sirvieron como centros de difusión de las ideas por medio de publicaciones, conferencias y museos. De acuerdo con Poskett (2019), la frenología comenzó a ser concebida como una ciencia global y esas sociedades actuaban como centros de conexión e intercambio de ideas y materiales. Las aplicaciones de la frenología como disciplina capaz de reformar la sociedad abarcaban desde el diagnóstico de criminales hasta la justificación de la esclavitud.

Aun siendo presentada como una ciencia reformadora de la sociedad, la frenología estaba fuertemente ligada a un carácter innatista o de determinismo biológico. Si bien era posible para un individuo, según algunos frenólogos, desarrollar aquellos órganos menos "potentes" (Sysling, 2018), ese desarrollo estaba limitado por la propia naturaleza, especialmente en lo relativo a la raza. De acuerdo con Poskett $(2019$, 21), la Sociedad Frenológica de Edimburgo tuvo como uno de sus objetivos "cartografiar la historia y geografía de la humanidad". Por lo tanto, se creía que a través del estudio frenológico sería posible determinar las características psicológicas de las diferentes naciones o de las diferentes razas. El componente racial y la jerarquización en la práctica frenológica tiene que ver con el grado de desarrollo que presentan en las razas los órganos que se corresponden con funciones específicas del ser humano, como, por ejemplo, la atención, el razonamiento, el ingenio o la bondad (Sakalauskaité-Juodeikienè, Eling, y Finger, 2017). La idea general compartida por los frenólogos era que el desarrollo de la región frontal determinaba una mayor inteligencia, pues en esa región se encontrarían los órganos correspondientes a las facultades psicológicas superiores características del ser humano, mientras que el desarrollo de la región posterior sería más propio de los animales. En base a ese supuesto, era sencillo establecer un paralelismo entre las razas inferiores y los animales, y justificar la inferioridad de unas razas sobre otras.

A tenor de ese paralelismo resulta interesante el caso de Eustache Belin, un esclavo negro haitiano que fue exhibido por los frenólogos como un individuo excepcional en su raza por haber rechazado la violencia de los otros esclavos hacia los esclavistas en las colonias de las Antillas francesas. Ese comportamiento excepcional tenía que responder al desarrollo del órgano de la benevolencia y, tras realizar un molde de su cabeza en París, así lo confirmaron los frenólogos (Poskett, 2019, 59). Ese molde fue desde entonces utilizado como la excepción 
que confirma la regla: Belin era excepcional porque no presentaba el carácter "negro" causado por una retracción de la región frontal del cráneo, causa de la inteligencia deficiente de la raza negra (Poskett, 2019, 61).

La divulgación de las tesis frenológicas y la popularización de la disciplina por medio de la publicidad en la prensa, las conferencias, los museos y gabinetes y las demostraciones públicas sirvieron para transmitir una visión naturalizada de la raza, que a lo largo de gran parte del siglo XIX sirvió como apología del colonialismo, la esclavitud y la segregación racial.

\subsection{Craneometría}

A pesar de la popularidad de la frenología hasta finales del siglo XIX (Sysling, 2018), en el ámbito científico el estudio de la forma del cráneo se vio en cierto momento como insuficiente. La medición del cráneo o craneometría comenzó a ser desarrollada en el siglo XVIII, siendo los dos principales pioneros de la disciplina Johann Friedrich Blumenbach y Peter Camper. Blumenbach utilizó como patrón de comparación de los cráneos la norma verticalis, es decir, el estudio del cráneo visto desde arriba. Camper llamó la atención sobre una diferencia esencial entre el ángulo facial de los "Europeos" y de los "Negros": estos últimos presentan un grado de "prognatismo" mayor, es decir, su mandíbula sobresale más (Jahoda, 2009).

Samuel George Morton, conocedor de las teorías de Blumenbach, comenzó a coleccionar cráneos en 1830 para mostrar las diferencias entre las razas que este último había identificado (aunque lo hizo sin basarse en la norma vertical (Jahoda, 2009, 38)): Caucásico o blanco, Mongólico o amarillo, Americano o rojo, Malayo o pardo y Etíope o negro. Morton introdujo la medición de la capacidad interna del cráneo como medida complementaria a las propuestas por Blumenbach y Camper. Asumió la hipótesis de que la capacidad interna del cráneo estaba directamente relacionada con el tamaño del cerebro y que este, a su vez, estaba directamente relacionado con la inteligencia. Por lo tanto, aquellas razas con una mayor capacidad craneal tendrían un cerebro más desarrollado, siendo así superiores. En base a sus medidas concluyó que la jerarquía racial básica correcta era la siguiente: Caucásico - Mongoloide - Negroide (Lieberman, 2001, 69).

El nexo entre la frenología y la craneometría alcanzó, probablemente, su punto álgido con la colaboración entre Morton y George Combe, el frenólogo británico más destacado y fundador de la Sociedad Frenológica de Edimburgo. Combe asistió a Morton en la elaboración de la más importante obra de este, Crania Americana (1839), dedicada al estudio de cráneos de nativos americanos. La obra incluye un apéndice de medidas frenológicas elaborado por Combe a partir de los datos de Morton, evaluando así el conjunto de facultades calificadas como "Sentimientos" y como "facultades Intelectuales" de la raza americana (Fabian, 2010, 92). 
Los datos recogidos por Morton en su colección de más de 1000 cráneos tuvieron mucho impacto en la época y otros autores los utilizaron para consolidar la jerarquización racial. Josiah C. Nott y George R. Gliddon (1854) desarrollaron la idea de que las razas se encontrarían, al igual que los animales, distribuidas geográficamente en función de sus características, pues así fueron creadas (como Morton, defendían la teoría poligenista). Nuevamente, uno de los argumentos principales es que la ciencia craneológica permite reconocer una desigualdad intelectual entre razas que es un hecho, así como reconocer que hay similitudes morfológicas entre las razas inferiores y el reino animal.

Por otra parte, en Francia, en plena revolución darwinista, Paul Broca y los científicos de su escuela contribuyeron considerablemente a los estudios craneométricos. La craneometría parecía la herramienta perfecta para cuantificar la evolución. Broca incorporó a su lenguaje técnico una distinción introducida por Anders Adolf Retzius que posteriormente sería ampliamente utilizada en las comparativas de cráneos: la distinción entre dolicocéfalo (cráneo alargado) y braquicéfalo (cráneo ancho). Retzius estipuló que los cráneos dolicocéfalos eran propios de los europeos modernos (Giménez-Roldán, 2016), correlacionando así la dolicocefalia con un mayor desarrollo intelectual y psicológico al abrigo de la teoría evolucionista. En la distinción dolicocefalia/braquicefalia persiste la hipótesis frenológica que afirma que el desarrollo de la región frontal implica una inteligencia superior.

En la elaboración de su teoría de las razas, Broca articuló las siguientes variables: desarrollo de las regiones frontal y parietal, índice craneal y la posición del foramen magnum. Las dos primeras variables determinan la dolicocefalia o braquicefalia, mientras que la explicación de la tercera apela al proceso evolutivo del ser humano. La hipótesis evolutiva de Retzius sobre la dolicocefalia chocó con los resultados que obtuvo Broca en sus mediciones de cráneos contemporáneos franceses, lo cual le llevó a considerar que el planteamiento de Retzius era prejuicioso, pues era sueco y en esa población predomina ampliamente la dolicocefalia.

El caso de la posición relativa del foramen magnum, por otro lado, pone en evidencia las tácticas retóricas de Broca para salvar sus ideas preconcebidas. Broca consideró que en las razas inferiores el foramen magnum debería encontrarse en una posición más posterior, pues es como se da en los primates. Dada la teoría de la evolución y el hecho del bipedismo, sería razonable concluir que el agujero debería encontrarse en una posición más central para permitir andar erguidos. $\mathrm{Al}$ realizar mediciones en cráneos de individuos negros, constató que el agujero se encontraba, efectivamente, en una posición más posterior y afirmó la tendencia de la conformación "del Negro, en este respecto como en muchos otros, a aproximarse a la del mono" (Gould, 1996, 133). Sin embargo, en sus mediciones incluyó la proyección facial y al reevaluar la posición relativa del agujero con respecto al cráneo obtuvo como resultado una posición más anterior. Ante la dificultad de salvar esta contradicción, pues otros parámetros (el prognatismo 
facial, por ejemplo) probaban, según Broca, la inferioridad de la raza negra, apeló a la distinción entre las regiones anterior y posterior del cráneo. La conclusión es idéntica a la de la frenología: la raza negra tiene más desarrollada la región posterior, lo cual prueba su inferioridad por su semejanza con los simios, mientras que la raza blanca presenta cráneos más desarrollados en la región anterior, muestra de su mayor desarrollo intelectual y psíquico.

\subsection{Demografía racial}

Tanto desde la frenología como desde la craneometría hubo la pretensión de explicar científicamente la distribución de las razas en el mundo e identificar las diferencias entre las diferentes naciones. El ejemplo del rechazo de Broca de la hipótesis de Retzius por ser prejuiciosa es ejemplo de ello, pues el problema realmente era que, mayoritariamente, los cráneos contemporáneos franceses eran braquicéfalos. También es cierto que Retzius asignó un tipo de cráneo a las naciones europeas. Las naciones dolicocéfalas (Suecia, Noruega, Dinamarca...) fueron catalogadas como "Nórdicas", mientras que las braquicéfalas (Hungría, Turquía, Finlandia...) eran más bien "una extraña mezcla” y el propio Retzius consideró que estas eran inferiores a aquellas (Jahoda, 2009, 41).

Sin embargo, se considera que el padre de la demografía racial es Joseph Arthur de Gobineau (1915), quien desarrolló a mediados del siglo XIX la potente idea de la superioridad de la raza aria sobre las demás razas. La frenología en esta disciplina, especialmente en los desarrollos de finales de siglo, no desempeña ya un papel relevante; son los datos de la craneometría los que aportan el criterio central para comparar las razas. Los criterios de referencia en esta disciplina fueron la forma del cráneo (dolicocefalia/braquicefalia), la estatura de los individuos y el color de piel y de cabello y ojos. Gobineau tuvo en cuenta en la elaboración de su teoría las propuestas craneométricas de Blumenbach y Morton, entre otros, pero consideró que esos resultados no eran concluyentes, por lo que tuvo que apoyarse en otras características físicas. Las tres razas reconocidas por Gobineau (blanca, amarilla y negra) se diferencian por esas características físicas e intelectuales. Mantuvo el análisis respectivo al desarrollo de las regiones frontal y posterior del cráneo, así como las conclusiones de los otros autores, pero complementó todos esos rasgos observables con la revisión de la historia de las grandes civilizaciones, probando que estas solo pudieron ser iniciadas por la raza blanca (concretamente por su estirpe aria), aunque hubiera mestizaje de por medio, pues este sería algo inevitable a expensas de la superioridad innata de la raza blanca. Raza y civilización, por tanto, aparecen unidas en la propuesta de Gobineau.

La cuestión aria fue desarrollada posteriormente por Georges Vacher de Lapouge (1899) quien se propuso explicar la historia y el momento actual de Europa por medio de las tres razas europeas: Homo europaeus, Homo alpinus y Homo mediterraneus. El individuo de la primera de ellas sería el ario: dolicocé- 
falo, de alta estatura y de piel, cabello y ojos claros. Lapouge, influenciado por el darwinismo social, incluyó también en las variables rasgos psicológicos como la valentía o el espíritu conquistador del ario, cualidades dadas por la propia naturaleza que lo consagraban a gobernar y ocupar los estratos superiores de la sociedad.

El darwinismo social fue determinante para dar forma a la "antroposociología", la disciplina desarrollada por Lapouge (1909) que recogía una serie de leyes fundamentales que regirían la distribución geográfica y estratigráfica de las razas tomando siempre como indicador de referencia el índice cefálico, pues "el cráneo y la psicología del individuo están influenciados de un modo correlativo por la estructura cerebral" (Lapouge, 1909, 178). Las leyes de la antroposociología abordan cuestiones económicas, geográficas y de organización social correlacionando el índice cefálico con la distribución de los individuos. Por ejemplo, la ley de distribución de la riqueza estipula que "en los países mixtos Europaeus-Alpinus, la riqueza crece en razón inversa a índice cefálico" (Lapouge, 1909, 178), mientras que la ley de estratificación estipula que "el índice cefálico disminuye y la proporción de dolicocéfalos aumenta desde las clases inferiores a las clases superiores en cada localidad" (Lapouge, 190, 206). Todas estas leyes justificarían apoyándose en el criterio científico craneométrico del índice cefálico la superioridad de la raza aria. La jerarquía social de Vacher de Lapouge prácticamente equipara la raza con la clase social, pues la movilidad social es extremadamente limitada; el orden social jerárquico está fijado por la naturaleza misma.

William Z. Ripley (1915) desarrolló también una teoría sociológica sobre las razas de Europa desde una perspectiva darwinista social. Redefinió el concepto de raza para distinguir las peculiaridades transmitidas por herencia de los rasgos mentales que responden al ambiente. Mantuvo los criterios centrales de Gobineau y Lapouge, pero otorgó todo el poder al índice cefálico, considerándolo la "característica racial fundamental" (Ripley, 1915, 305). Aunque Ripley insistió mucho en la importancia del ambiente y de la selección sexual, y criticó la cuestión de la supuesta raza aria por darse en ella un cruce de categorías entre raza, lenguaje y cultura (lo ario sería lingüístico y no tiene por qué coincidir con las razas), sí que se observan en su obra numerosos sesgos, especialmente en lo relativo a características psicológicas. Esos sesgos son, realmente, los prejuicios comunes a la mayoría de los científicos raciales de la época. Ripley distinguió tres razas europeas: teutónica, alpina y mediterránea; todas ellas se distinguen por los criterios antropométricos ya expuestos, pero también (y nuevamente) por características psicológicas. La raza teutónica, por ejemplo, es “móvil” y por eso tiende a conquistar e invadir territorios, mientras que la raza alpina, confinada principalmente en regiones montańosas y dedicada principalmente a la agricultura, es sedentaria y conservadora (Ripley, 1915, 550). Asimismo, afirma que en la Asia de los "mongoles de ojos rasgados" se da una "cultura nómada e imperfecta” (Ripley, 1915, 338). 


\subsection{Antropología criminal}

Partiendo de la teoría de la evolución y del estudio craneométrico y antropométrico de los individuos, complementado con una perspectiva psicológica, Cesare Lombroso desarrollo la disciplina de la antropología criminal. Lombroso propuso el innatismo y la heredabilidad de la criminalidad, por lo que sería posible identificar a aquellos individuos predispuestos a ella por una serie de indicadores físicos o psicológicos, así como identificar linajes completos de criminales. Apoyándose en la teoría de la evolución, sostuvo que los criminales constituían recesos evolutivos identificables antropométricamente. Habría en los criminales un atavismo físico y mental que permitiría compararlos con lo que Lombroso denominaba "salvajes".

En Criminal Man (2006), su obra más relevante, Lombroso expone todos los parámetros que permiten la identificación de los criminales: medidas craneométricas y antropométricas, el clima, las emociones y la raza, entre otras. En cuanto a las medidas craneométricas, Lombroso tomó como referencia la medida de la circunferencia del cráneo, el estado de las suturas y la presencia de anomalías estructurales. En términos generales, los resultados que obtuvo indicaban una correlación entre la criminalidad y los cráneos pequeños y braquicéfalos, lo cual confirmaba la hipótesis de que la dolicocefalia y un cráneo mayor son características de los individuos más avanzados o evolucionados. Los cráneos de los criminales en raras ocasiones presentaban suturas normales, pero, como evidencia del carácter innato de la criminalidad, presentaban a menudo anomalías estructurales presentes desde el nacimiento. Corroborando lo atávico del criminal, Lombroso afirmó que no podía evitar "señalar lo estrechamente que se corresponden con características observadas en cráneos normales de las razas coloreadas e inferiores" (Lombroso, 2006, 48). Entre esas anomalías estructurales se encontraban características tradicionalmente atribuidas a las razas inferiores como la mandíbula grande y las cejas salientes, pero Lombroso identificó también un mayor tamaño de la foseta occipital media, similar al de las razas más bajas de los monos y que, de acuerdo con Lombroso, se observa con frecuencia en los neozelandeses y los aymaras (Lombroso, 2006, 305). Esta última característica se ajusta a la hipótesis presente en las disciplinas expuestas anteriormente sobre el desarrollo frontal o posterior del cráneo, pues el hundimiento de la fosa occipital (en la región posterior del cráneo) se observa en algunas especies animales.

Lombroso, además, también elaboró lo que se podría caracterizar como un atlas de la criminalidad y sus conclusiones fueron recogidas, por ejemplo, por Ripley (1915). En su "demografía criminal" centrada principalmente en Italia, Lombroso (1901) relacionó tipos de crimen con las distintas razas. Identificó zonas como Sicilia en las que la influencia árabe explicaría a la perfección el alto ratio de bandolerismo sin sentir horror o repulsión por esa práctica como sucedería "en grupos con una mayor proporción de sangre aria" (Lombroso, 2006, 118). En los mapas presentados por Lombroso es común encontrar unos mayores índi- 
ces de criminalidad en el sur de Italia, lo cual casaba con su creencia de que el sur era racialmente inferior al norte debido a que los italianos del sur tenían sangre africana y árabe (Lombroso, 2006, 408).

A pesar de su insistencia en el carácter innato de la criminalidad, Lombroso también tuvo en consideración la influencia del ambiente sobre esta. Su "geografía del crimen" recogida en numerosos mapas (2006), pretendía mostrar la importancia de la raza en los tipos de crímenes (en el caso de los árabes o los albanos, la criminalidad está relacionada con el bandolerismo) como la del clima. Nuevamente, en el sur de Italia hay unos mayores índices de criminales por su inferioridad racial, pero las regiones de esa zona son también las más calurosas y con menos densidad de población. La combinación de estos tres parámetros — raza, clima y densidad de población — queda bien recogida en la siguiente afirmación: "las tasas de asesinato aumentan en regiones de densidad mínima, que en Italia corresponden a las partes más calurosas y barbáricas de la península y, más a menudo, sus islas" (Lombroso, 2006, 316).

\subsection{Pruebas de medición de la inteligencia}

A finales del siglo XIX la craneometría fue mayoritariamente abandonada en beneficio de la medición de la inteligencia. El caso de Alfred Binet ejemplifica cómo la falta de conclusividad de la craneometría derivó en un abandono de la disciplina, pues al realizar mediciones craneológicas no encontró diferencias significativas. Dada la escasa utilidad de las medidas craneométricas, Binet se propuso estudiar lo psicológico de los individuos y junto con Théodore Simon desarrolló una escala de medición de la inteligencia con el objetivo de que esta sirviera como apoyo para la educación de los niños. De acuerdo con Gould (1996), Binet no cosificó la inteligencia, es más, mostraba preocupación por las consecuencias sociales que ello pudiera acarrear, y, por ello y aceptando la exposición de Gould, sus estudios no serán aquí tratados.

Una vez superados los intentos de identificar y jerarquizar las razas apelando a características físicas medibles, la inteligencia cosificada como una entidad medible y genética tomó el relevo en ese proyecto. La cosificación de la inteligencia está estrechamente relacionada con el darwinismo social y las propuestas eugenistas de las últimas décadas del siglo XIX y la teoría hereditarista de la inteligencia está necesariamente ligada a la cuestión de la raza, pues diagnosticaría las diferencias intelectuales sobre la base de la biología presuponiendo una disposición diferente en función de la raza. La teoría hereditarista de la inteligencia y su articulación a través de numerosos desarrollos y aplicaciones de pruebas de medición de la inteligencia sirvió para jerarquizar las sociedades y las culturas.

Fue en Estados Unidos, probablemente debido a la situación social del propio país tras la abolición de la esclavitud, inmerso en una fuerte segregación racial, donde más se exploraron las consecuencias racistas de las pruebas de medición de 
la inteligencia. Henry Herbert Goddard fue el primero en popularizar la escala de Binet-Simon en Estados Unidos asumiendo que los resultados que se obtenían en ella remitían a una entidad innata singular. Habiendo desarrollado su propia escala unilineal que medía la "deficiencia mental", concluyó que esta se ajustaba a los patrones de herencia mendeliana. En su jerarquización social, los inteligentes serían aquellos hombres que gobernarían por derecho, mientras que los otros dos estratos de la sociedad estarían conformados por meros tontos (la masa de trabajadores) y los morons (término acuñado por Goddard), que serían los criminales, las prostitutas y los alcohólicos, entre otros. En plena década de 1910, con una intensa migración a Estados Unidos, Goddard puso en marcha un estudio masivo de la inteligencia tomando como sujetos a los migrantes que llegaban a la Isla Ellis (Nueva York). El alto porcentaje que obtuvo de "débiles mentales" le llamó la atención y tuvo que ajustar su propia escala, quizás demasiado exigente. Rechazó la consideración de otras variables que pudieran influir en resultados tan negativos por parte de personas que llegaban a un país desconocido, que se enfrentaban a una lengua desconocida, que habían viajado durante semanas en malas condiciones, entre otros factores. Como resultado de su experimento, el número de deportaciones en los ańos siguientes aumentó considerablemente (Gould, 1996).

Tras la entrada de Estados Unidos en la Primera Guerra Mundial, Robert Mearns Yerkes encabezó una comisión dedicada a la elaboración de pruebas de medición de la inteligencia para evaluar a los reclutas del ejército estadounidense. Para ello se desarrollaron tres tipos de pruebas en función del nivel educativo de los individuos: una prueba escrita, una prueba pictórica y un examen individual basado en la escala de Binet. El rango militar al que podría acceder el recluta estaba determinado por el resultado obtenido en la prueba correspondiente. Los datos generales arrojados por el conjunto de pruebas realizadas determinaron tres cuestiones: (i) la media blanca nacional era muy baja, (ii) era posible clasificar a los inmigrantes europeos en función de su país de origen, y (iii) la raza negra se encontraba siempre en el nivel más bajo.

A través de los resultados de las pruebas de medición de la inteligencia, tanto Yerkes como Carl Brigham intentaron elaborar algo parecido a la demografía racial, prestando especial atención a la raza negra. Ambos, interesados por las diferencias en los resultados entre los negros del norte del país con respecto a los del sur, llegaron a la conclusión de que los negros más inteligentes habían migrado hacia el Norte (Gould, 1996), resaltando Brigham la mayor cantidad de sangre blanca en estos, un factor, desde una perspectiva, relevante para el nivel de inteligencia. Brigham, además, explicó las considerables diferencias en los resultados de las pruebas realizadas a los inmigrantes de diferentes oleadas de migración tomando como referencia la propuesta de Lapouge de las tres razas puras europeas (López Cerezo y Luján López, 1989). Las oleadas de inmigración más recientes eran, principalmente, de europeos del sur, centro y este, mientras que 
en las anteriores hubo un importante flujo de europeos de los países del norte. Asumiendo la teoría racial de Lapouge, Brigham excluyó la relevancia del factor ambiental en los resultados.

\section{Discusión}

Calificar a las disciplinas estudiadas como pseudocientíficas desde la actualidad sin tener en cuenta el contexto en el que fueron desarrolladas y promovidas puede llegar a ser incluso injusto. Ello porque es preciso reconocer las diferencias instrumentales, metodológicas y cognoscitivas entre esta época y aquella. Sin embargo, el análisis planteado en esta investigación sostiene el juicio de pseudocientificidad de las disciplinas tomando como núcleo una serie de cuestiones externas (valores, prejuicios, ideología) que comprometen la investigación científica en todas sus partes. Esto es labor de la perspectiva diacrónica. Desde esta perspectiva, además, es posible aplicar una serie de criterios como los propuestos por Hansson (2013) para identificar si una disciplina es pseudocientífica. Unos criterios como los que propone el autor, aun no siendo exhaustivos, son útiles como guía para someter a crítica las disciplinas. Por otro lado, desde una perspectiva sincrónica, se ha pretendido mostrar cómo estas disciplinas eran aceptadas como científicas hasta que surgía un momento de inflexión en el que eran abandonadas y sustituidas por otras.

El concepto de raza presente en todas ellas es sumamente problemático. Está construido a partir de una serie de valores, prejuicios y componentes sociales que se introducen en el proceso de indagación científica contaminándolo. Dado que se toma como referencia un periodo temporal de alrededor de 100 años no es posible sancionar que el concepto de raza se corresponda con un tipo de sociedad concreto, como podría ser la sociedad victoriana. Al contrario, se observa cómo, a pesar de haber cambios en las sociedades, el concepto de raza continúa siendo un concepto cargado de valores y prejuicios que se mantiene al margen de esos cambios.

El recorrido de la idea de raza en las disciplinas muestra cómo los científicos no buscaban evidencia, sino confirmar sus creencias, justificar prejuicios y preservar cierto statu quo. La idea de raza presente en ellas es una idea que permanece fija e inmutable: unas razas son superiores a otras y, concretamente, la raza blanca es superior a las demás, mientras que la raza negra es siempre inferior. Cuando la evidencia científica no apoyaba esa idea o cuando los métodos de la disciplina no daban más de sí, se recurría a la revisión metodológica propiciando el cambio de disciplinas.

El discurso racial forma parte de lo cotidiano e inevitablemente se introduce en el ámbito científico. En las últimas décadas se puede observar cómo la cientifización y biologización de la raza sigue siendo una cuestión candente que tomó especial fuerza a través de, entre otras, la sociobiología. La problemática de las 
disciplinas aquí expuestas no descansa, como he mencionado, en que considerasen que la raza era una realidad biológica, sino que su dirección de investigación y sus conclusiones descansaban sobre una idea de raza que responde a una serie de creencias y prejuicios presentes en la sociedad. El problema es, por tanto, cómo la búsqueda de las bases biológicas de la raza depende directamente de una concepción social de la raza que es vertida sobre la ciencia y permea todo el proceso de indagación científica. En estas disciplinas se observa un concepto racista y una constante naturalización de lo social. La carga de valores en estas disciplinas es muy alta y rompe con la pretendida objetividad tras la que se escudaban aquellos científicos.

Aceptando los criterios generales y amplios propuestos por Hansson (2013), se puede concluir que más allá de la evidencia científica disponible en la época, no comparable con la actual, todas estas disciplinas sí pertenecían al dominio científico, pero su fiabilidad estaba comprometida por la alta carga de valores y constituyen un claro ejemplo de doctrina desviada.

\section{Conclusiones}

En esta investigación se ha pretendido mostrar cómo una misma idea de raza fue utilizada como hilo conductor durante todo un siglo, pero esa idea ha tenido una trayectoria mucho más amplia en la ciencia, tanto antes como después del periodo delimitado para este estudio. La exposición de todas estas disciplinas arroja luz sobre el modo en que las creencias populares o los prejuicios se introducen en el discurso científico. La profundización en estas cuestiones es prometedora. A continuación, se plantean algunos aspectos que han sido considerados de manera sucinta y que merecerían una mayor atención, así como otros aspectos relacionados con la cuestión aquí tratada y que son de gran interés.

En primer lugar, el rol de la autoridad epistémica con respecto a la raza plantea un asunto doble de suma importancia: el uso de la autoridad epistémica que otorga la ciencia para justificar creencias, prejuicios y preservar un statu quo y la relevancia de la autoridad epistémica cuando los datos que arroja la ciencia no encajan con las creencias. Las disciplinas expuestas ejemplifican el primer aspecto de la autoridad epistémica, mientras que, con respecto al segundo aspecto, cabe preguntarse qué sucede, por ejemplo, cuando la ciencia aporta información que lleva a concluir que no hay razas biológicas, pero el concepto de raza y las clasificaciones raciales forman parte del discurso ordinario.

En segundo lugar, y en relación con la cuestión anterior, el estudio de la idea de raza en la ciencia ofrece muchas posibilidades para la crítica filosófica desde una perspectiva de Ciencia, Tecnología y Sociedad. La cuestión de la raza ejemplifica de una manera muy evidente cómo esas tres dimensiones están íntimamente relacionadas y por qué deben ser estudiadas en profundidad para aproximarse lo máximo posible a la obtención de respuestas satisfactorias. En las 
últimas décadas la idea de raza ha seguido muy presente en la ciencia y lo normal es esperar que siga siendo así en el futuro. Existan o no las razas biológicas, ¿qué supone eso para la sociedad? ¿Cómo influye el discurso popular en el desarrollo de la ciencia? Y, un interrogante de sumo interés en relación con las propuestas naturalistas, ¿es concebible la aceptación de la existencia de razas biológicas sin que ello derive en el racismo?

En tercer lugar, la cuestión de la raza en ciencia está íntimamente ligada a los estudios de ciencia y género. Estos estudios advierten de los sesgos y la limitada perspectiva que aqueja a la ciencia tradicional y a los estudios sobre la ciencia tradicionales. Las perspectivas feministas no limitan su labor a la cuestión de la mujer y del género, sino que ponen en relieve la necesidad de tener en cuenta otras variables como la clase, la raza o la cultura. Por ello, no solo es una cuestión interesante por sí misma, sino que la investigación sobre ella sirve para entablar una conversación interesante y ya iniciada con las perspectivas feministas de la ciencia.

Por último, el análisis del concepto de raza debe ser abordado desde una perspectiva filosófica teórica. Las reflexiones vertidas desde la Filosofía de la biología acerca de las clases naturales, las esencias y la taxonomía hallan un importante foco de interrogantes en la cuestión de la raza. Es preciso estudiar qué se entiende por raza en el discurso científico y evaluar las diferentes propuestas filosóficas de conceptualización de la raza: naturalismo racial, constructivismo racial y escepticismo racial.

\section{Referencias bibliográficas}

Cantor, Geoffrey N. (1975). Phrenology in Early Nineteenth-Century Edinburgh: An Historiographical Discussion. Annals of Science, 32(3), 195-218.

Fabian, Ann (2010). The Skull Collectors: Race, Science, and America's Unburied Dead. Chicago: The University of Chicago Press.

Giménez-Roldán, Santiago (2016). Paul Broca’s search for Basque skulls: The full story. Journal of the History of the Neurosciences, 25(4), 371-385.

Gobineau, Arthur de (1915). The Inequiality of Human Races. London: William Heinemann.

Goode, Erich (2013). Paranormalism and Pseudoscience as Deviance. En M. Pigliucci y M. Boudry (Eds.), Philosophy of Pseudoscience (pp. 145-164). Chicago: The University of Chicago Press.

Gómez Rodríguez, Amparo (2004). La estirpe maldita: la construcción cientifica de lo femenino. Madrid: Minerva Ediciones.

Gould, Stephen Jay (1996). The Mismeasure of Man (Revised and Expanded). Nueva York: W. W. Norton \& Company. 
Gregory, Jane y Miller, Steven (1998). Science in Public: Communication, Culture and Credibility. Cambridge: Basic Books.

Hansson, Sven O. (2013). Defining Pseudoscience and Science. En M. Pigliucci y M. Boudry (Eds.), Philosophy of Pseudoscience (pp. 61-77). Chicago: The University of Chicago Press.

Jahoda, Gustav (2009). Intra-European Racism in Nineteenth-Century Anthropology. History and Anthropology, 20(1), 37-56.

Kaufman, Matthew H. (1998). The Edinburgh Phrenological Debate of 1823 Held in the Royal Medical Society. Journal of Neurolinguistics, 11(4), 377-389.

Lapouge, Georges Vacher de (1899). L'Aryen: Son Role Social. París: Albert Fontemoing.

Lapouge, Georges Vacher de (1909). Race et Milieu Social : Essais d'Anthroposociologie. Paris: Marcel Riviére.

Laudan, Larry (1983). The Demise of the Demarcation Problem. En R. S. Cohen y L. Laudan (Eds.), Physics, Philosophy and Psychoanalysis. Boston Studies in the Philosophy of Science (vol. 76). Dordrecht: Springer.

Lieberman, Leonard (2001). How "Caucasoids" Got Such Big Crania and Why They Shrank. Current Anthropology, 42(1), 69-95.

Lombroso, Cesare (1911). Crime: Its Causes and Remedies. London: William Heinemann.

Lombroso, Cesare (2006). Criminal Man. Durham: Duke University Press.

López Cerezo, José Antonio y Luján López, José Luis (1989). El artefacto de la inteligencia. Barcelona, España: Anthropos.

Lyons, Sherrie (1998). Science or Pseudoscience: Phrenology as a Cautionary Tale for Evolutionary Psychology. Perspectives in Biology and Medicine, 41(4), 491-503.

Nott, Josiah C. y Gliddon, George R. (1854). Types of Mankind. Philadelphia: Lippincott, Grambo \& Co.

Ripley, William Z. (1915). The Races of Europe: A Sociological Study. New York: D. Appleton \& Company.

Sakalauskaitè-Juodeikiené, Eglè, Eling, Paul y Finger, Stanley (2017). The reception of Gall's organology in early-nineteenth-century Vilnius. Journal of the History of the Neurosciences, 26(4), 385-405.

Shapin, Steven (1975). Phrenological Knowledge and the Social Structure of Early Nineteenth-Century Edinburgh. Annals of Science, 32(3), 219-243.

Shermer, Michael (2013). Science and Pseudoscience: The Difference in Practice and the Difference it Makes. En M. Pigliucci y M. Boudry (Eds.), Philosophy of Pseudoscience (pp. 121-144). Chicago: The University of Chicago Press.

Sysling, Fenneke (2018). Science and self-assessment: phrenological charts 1840-1940. British Society for the History of Science, 51(2), 261-280. 
Thomassen, Jacob L. y Beierholm, Simon (2020). Franz Joseph Gall came to Copenhagen, and for a brief moment the brain was the talk of the town. Journal of the History of the Neurosciences, 29(1), 48-59.

Thurs, Daniel P. y Numbers, Ronald L. (2013). Science, Pseudoscience, and Science Falsely So-Called. En M. Pigliucci y M. Boudry (Eds.), Philosophy of Pseudoscience (pp. 121-144). Chicago: The University of Chicago Press.

Van Wyhe, John (2004). Was Phrenology a Reform Science? Towards a New Generalization for Phrenology. History of Science, 42, 313-331.

Whitaker, Harry y Jarema, Gonia (2017). The split between Gall and Spurzheim (18131818). Journal of the History of the Neurosciences, 26(2), 216-223. 